\title{
The Long Non-Coding RNA LncRNA8975-1 is Upregulated in Hypertrophic Scar Fibroblasts and Controls Collagen Expression
}

\author{
Jun Lia Ling Chen ${ }^{a}$ Chunyu Cao ${ }^{b}$ Hui Yan ${ }^{a}$ Bei Zhou ${ }^{a}$ Yanli Gao ${ }^{a}$ Qian Lia \\ Jingyun $\mathrm{Li}^{\mathrm{a}}$
}

\begin{abstract}
aState key Laboratory of Reproductive Medicine, Department of Plastic\&Cosmetic Surgery, Maternal and Child Health Medical Institute, Obstetrics and Gynecology Hospital Affiliated to Nanjing Medical University, Nanjing, bepartment of Obstetrics, Maternal and Child Health Care Hospital of Nantong, Nantong, China
\end{abstract}

\section{Key Words}

Long non-coding RNA • LncRNA8975-1 • Collagen • Hypertrophic scar • Fibroblasts

\begin{abstract}
Background/Aims: Long non-coding RNAs (IncRNAs) are thought to play crucial roles in human diseases. However, the function of IncRNAs in hypertrophic scar formation remains poorly understood. Methods: In this study, we investigated the expression of IncRNA8975-1 in hypertrophic scar tissues and fibroblasts by quantitative reverse transcription PCR (qRT-PCR). To investigate its function, overexpression and knockdown of IncRNA8975-1 were performed using lentivirus infection and Stealth RNAi transfection, respectively. Cell proliferation was detected by CCK- 8 assay. The protein levels of collagens and alpha-smooth muscle actin ( $\alpha$-SMA) were analysed by western blot. Results: We found that IncRNA8975-1 was overexpressed in hypertrophic scar tissues and dermal fibroblasts. Overexpression of IncRNA8975-1 inhibited cell proliferation and reduced the protein expression levels of COL1A2, COL1A1, COL3A1 and $\alpha$-SMA in hypertrophic scar fibroblasts, whereas knockdown of IncRNA8975-1 had the opposite effect. Conclusion: Our results show that the long non-coding RNA IncRNA8975-1 is upregulated in hypertrophic scar fibroblasts; furthermore, it inhibits fibroblast proliferation and reduces collagen and $\alpha$-SMA expression. Further studies on the mechanisms regulated by IncRNA8975-1 would lead to a better understanding of the pathogenesis of hypertrophic scar formation.

\section{Introduction}

Hypertrophic scarring, a fibrotic disease, is a common and inevitable outcome of deep skin trauma or severe burn injury [1]. The overall incidence of hypertrophic scars for skin 


\section{Cellular Physiology Cell Physiol Biochem 2016;40:326-334 \begin{tabular}{l|l} 
and Biochemistry Published online: November 21, 2016 & $\begin{array}{l}\text { C } 2016 \text { The Author(s). Published by S. Karger AG, Basel } \\
\text { www.karger.com/cpb }\end{array}$
\end{tabular} \\ Li et al.: LncRNA Controls Collagen Expression}

trauma is $40 \%-70 \%$, whereas the incidence of burn scars is as high as $80 \%$ [2]. Hypertrophic scars often cause pain, pruritus and contractures and can significantly affect the physical and psychological health of patients. At present, although surgical excision, pressure therapy, steroid injections and radiation therapy are available to prevent and cure hypertrophic scars, none of these methods is optimal [3]. Therefore, understanding the mechanism of the formation of hypertrophic scars to identify new targets for treatment is highly desirable.

Emerging evidence has reported that activated fibroblasts with a higher capacity for cell proliferation and collagen synthesis contribute to the formation and development of hypertrophic scars [4, 5]. Hypertrophic scars are characterized by abundant alpha-smooth muscle actin ( $\alpha$-SMA)-producing myofibroblasts in conjunction with more type III collagen and type I collagen [6].

Long non-coding RNAs (lncRNAs) represent a class of transcripts longer than 200 nucleotides that lack protein coding potential [7]. Several studies have shown that lncRNAs play important roles in keloid formation [8], scleroderma [9], lung fibrosis [10], and other fibrotic disorders [11] by acting through diverse mechanisms. Recently, our group discovered that IncRNA8975-1 (TCONS_00013888 in UCSC hg38; also named NONHSAT122029 in NONCODEv4), an IncRNA located upstream of the COL1A2 gene on chromosome 7 (UCSC hg38, chr7:94278999-94392179, ChIPBase named it lncRNA8975-1), was overexpressed in regressive scars compared to mature scar tissues [12]. However, the functional role of lncRNA8975-1 in hypertrophic scars has not been documented.

In this study, fifteen hypertrophic scar samples and the corresponding matched normal skin tissues were used to perform qRT-PCR analysis. The results showed that lncRNA8975-1 was upregulated in hypertrophic scar tissues and fibroblasts, suggesting that there is an important correlation between lncRNA8975-1 expression and hypertrophic scarring. Using lentivirus infection and siRNA transfection, we found that lncRNA8975-1 affected hypertrophic scarring by inhibiting the proliferation of fibroblasts, which reduced the expression of collagens and $\alpha$-SMA. Our study provides clues for a new regulatory mechanism in the pathogenesis of hypertrophic scars.

\section{Materials and Methods}

\section{Ethics statement}

This study was approved by the Medical Ethics Committee of Obstetrics and Gynaecology Hospital Affiliated to Nanjing Medical University (No. [2013] 48). Patients attending our hospital for scar removal were provided information about the purpose of the study, and written informed consent was obtained from each participant.

Tissue samples

Fifteen hypertrophic scar (HS) tissues and their paired normal scar (NS) tissues were obtained from 15 different patients who were admitted to the Obstetrics and Gynaecology Hospital Affiliated to Nanjing Medical University for scar removal. A diagnosis of hypertrophic scarring was confirmed by routine pathological examination. The collected skin samples were divided into two fragments: one was used for the isolation and culture of fibroblasts, and the other was immediately frozen in liquid nitrogen for preparation of total RNA.

\section{Cell culture}

Cultures of hypertrophic scar fibroblasts (HSF) and normal skin fibroblasts (NSF) were established as previously described [13]. Explants were cultured in DMEM media (Invitrogen, Carlsbad, CA, USA) supplemented with $10 \%$ foetal bovine serum, $100 \mu \mathrm{g} / \mathrm{ml}$ streptomycin and $100 \mathrm{U} / \mathrm{ml}$ penicillin at $37^{\circ} \mathrm{C}$ in $5 \% \mathrm{CO}_{2}$. The fibroblasts in this study were used between their third and fifth passages.

Quantitative reverse transcription PCR assay

Total RNA was isolated from each tissue and corresponding cultured cells using TRIzol reagent (Invitrogen, Carlsbad, CA, USA). NanoDrop ND-1000 was used to measure RNA quantity. RNA was then 


\section{Cellular Physiology Cell Physiol Biochem 2016;40:326-334 and Biochemistry Published online: November 21, $2016 \begin{aligned} & \text { DOI: 10.1159/000452548 } 2016 \text { The Author(s). Published by S. Karger AG, Basel } \\ & \text { www.karger.com/cpb }\end{aligned}$ \\ Li et al.: LncRNA Controls Collagen Expression}

converted to cDNA using the First Strand cDNA Synthesis Kit (Invitrogen, Carlsbad, CA, USA) and a thermocycler (S1000, Bio-Rad, Berkeley, CA, USA). Quantitative reverse transcription PCR was performed using a SYBR Green qPCR mix (Invitrogen, Carlsbad, CA, USA) on an Applied Biosystems ViiA ${ }^{\text {TM }} 7$ Dx (Life Technologies, USA). The efficiency of amplification and detection by all primers was validated by determining the slope of Ct versus the dilution series. Transcript levels for IncRNA8975-1 were normalized to GAPDH cDNA levels using the standard curve method [14]. The primer sequences were as follows: IncRNA8975-1 (110 bp) forward primer, TCT GTG AGA AGA AGC AAT ACCA, reverse primer, CCT CAT TCA TGT CAT CTC CTCT; and GAPDH (226 bp) forward primer, GAA GGT GAA GGT CGG AGTC, reverse primer, GAA GAT GGT GAT GGG ATTTC. We used Primer-BLAST (https://www.ncbi.nlm.nih.gov/tools/primer-blast/index. cgi?LINK_LOC=BlastHome) to confirm the primer specificities for IncRNA8975-1 and GAPDH. Each value was the mean of the same sample from three independent experiments. Each value used for the boxplot was normalized to the average value of respective group.

\section{Lentivirus infection and Stealth RNAi transfection}

Lentiviral constructs carrying a 352 bp lncRNA8975-1 transcript (pGLV-H1-IncRNA8975-1-GFP/Puro) and an empty negative control vector (pGLV-H1-GFP/Puro) were obtained from GenePharma (Shanghai, China). Lentivirus infections were performed at $10 \mathrm{MOI}$. At $48 \mathrm{~h}$ post-transfection, the expression of the fluorescent protein GFP was observed under a fluorescence microscope (Observer.D1, Zeiss, Oberkochen, Germany). Images were collected using the microscope equipped with an AxioCam HRc camera and processed with Axiovision software (Zeiss, Oberkochen, Germany). Stealth RNAi ${ }^{\text {TM }}$ siRNA Negative Control and Stealth RNAi ${ }^{\mathrm{TM}}$ siRNA duplexes against IncRNA8975-1 were obtained by using the BLOCK-iT RNAi design program (Invitrogen, Carlsbad, CA, USA) and purchased from the ThermoFisher Scientific corporation. The sequences for the Stealth_151 duplexes are 5'-UGA CAA UCA AGU CAA CUA GAA CUG G-3' and 5'-CCA GUU CUA GUU GAC UUG AUU GUCA-3'. We used Nucleotide BLAST (https://blast.ncbi.nlm.nih.gov/Blast. cgi?PROGRAM=blastn\&PAGE_TYPE=BlastSearch\&LINK_LOC=blasthome) and the sequence alignment software Vector NTI (Invitrogen, Carlsbad, CA, USA) to test the specificity of the Stealth_151 duplexes for IncRNA8975-1. For reverse transfection, either Stealth RNAi ${ }^{\text {TM }}$ siRNA Negative Control (60 nM) or Stealth_151 siRNA (60 nM) was mixed with Lipofectamine RNAiMAX transfection reagent (Invitrogen, Carlsbad, CA, USA), and added to cells plated at $60 \%$ confluency in 6-well plates; these cultures were incubated at $37^{\circ} \mathrm{C}$ in $5 \% \mathrm{CO}_{2}$. At $96 \mathrm{~h}$ after either lentivirus infection or stealth RNAi transfection, the cells were harvested to detect the overexpression or knockdown efficiency via qRT-PCR.

\section{Western blot}

Total cellular protein was extracted using radioimmunoprecipitation assay (RIPA) buffer (Invitrogen, Carlsbad, CA, USA). The protein concentration was determined using a BCA kit (Bio-Rad). Proteins (15 $\mu \mathrm{g}$ ) were separated by SDS-PAGE and transferred to nitrocellulose membranes (Amersham, Chalfont, UK). The membranes were blocked in $5 \%$ non-fat dry milk followed by incubation with primary antibodies. Then, the membranes were incubated with either goat anti-rabbit IgG or goat anti-mouse IgG horseradish peroxidase (HRP)-conjugated secondary antibodies (1:2000, Abcam) and developed using chemiluminescence (ECL; Amersham). The primary antibodies used were as follows: anti-Ki67 rabbit polyclonal antibody (1:1000; ab15580, Abcam), anti-PCNA rabbit polyclonal antibody (1:500; ab18197, Abcam), anti-COL1A1 rabbit monoclonal antibody (1:1000; ab138492, Abcam), anti-COL1A2 rabbit polyclonal antibody (1:1000; ab96723, Abcam), anti-COL3A1 mouse monoclonal antibody (1:1000; ab6310, Abcam), anti-alpha-smooth muscle actin rabbit monoclonal antibody (1:1000; ab32575, Abcam), and anti-GAPDH mouse monoclonal antibody $(1: 2000 ;$ ab8245, Abcam). GAPDH was used as a loading control. The band intensity was quantified by densitometry of three independent experiments using Image J software (National Institutes of Health, Bethesda, MD, USA). The values used for the histogram were normalized to GAPDH and then normalized to control samples.

\section{Cell Counting Kit-8 (CCK-8) assay}

Cell proliferation was analysed using the CCK-8 kit (Vazyme Biotech co.,Itd, Nanjing, China). In brief, $24 \mathrm{~h}$ after either lentivirus infection or Stealth RNAi transfection, HSFs were seeded at $5 \times 10^{3}$ cells per well in 96-well plates with four replicates per condition. At 0, 24, 48 and $72 \mathrm{~h}, 10 \mu \mathrm{L}$ of CCK-8 solution was added to each well. After $1.5 \mathrm{~h}$ of incubation, the absorbance was measured at $450 \mathrm{~nm}$ on a microplate reader (Molecular Devices, Sunnyvale, CA, USA). 
Statistical analysis

Differences in the data between two groups were analysed using the SPSS 20.0 software package (SPSS, Chicago, IL, USA) with the Independent Samples $t$ Test. The data are presented as the means of results from three experiments with each experiment performed in duplicate. Statistical significance was defined as $P<0.05$.

\section{Results}

LncRNA8975-1 is overexpressed in hypertrophic scar tissue and fibroblasts

We previously reported that IncRNA8975-1 was upregulated in regressive scars compared to mature scartissue [12]. To determine whether lncRNA8975-1 was overexpressed in hypertrophic scars, we detected its expression in 15 hypertrophic scar tissues and the corresponding hypertrophic scar fibroblasts (HSF) compared to the matched normal skin and normal skin fibroblasts (NSF). The results revealed that lncRNA8975-1 mRNA was significantly overexpressed in hypertrophic scar tissues compared to normal skin tissues (Fig. 1A). Furthermore, lncRNA8975-1 mRNA expression in hypertrophic scar fibroblasts (HSF) was higher than in normal skin fibroblasts (NSF) (Fig. 1B).

Based on bioinformatics analyses, we found that lncRNA8975-1 is a transcript of 352 nucleotides and comprises 5 exons (NONHSAT122029 in NONCODEv4). LncRNA8975-1 contains no overlapping sequence with the transcripts from its neighbouring gene COL1A2

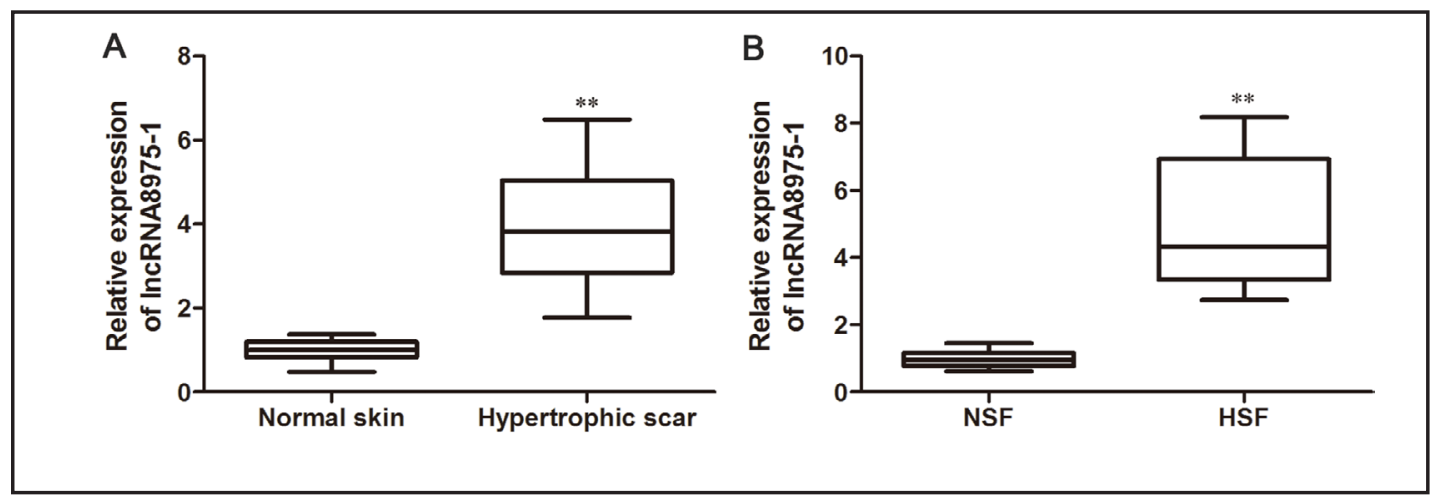

Fig. 1. Expression of IncRNA8975-1 in hypertrophic scar tissues and fibroblasts. (A) The expression of IncRNA8975-1 was validated in HS tissues $(\mathrm{n}=15)$ and their matched NS tissues $(\mathrm{n}=15)$ by qRT-PCR. (B) The expression of IncRNA8975-1 was validated in NSF $(\mathrm{n}=15)$ and HSF $(\mathrm{n}=15)$ by qRT-PCR. ${ }^{* *}$ Statistically significant difference at $P<0.01$.

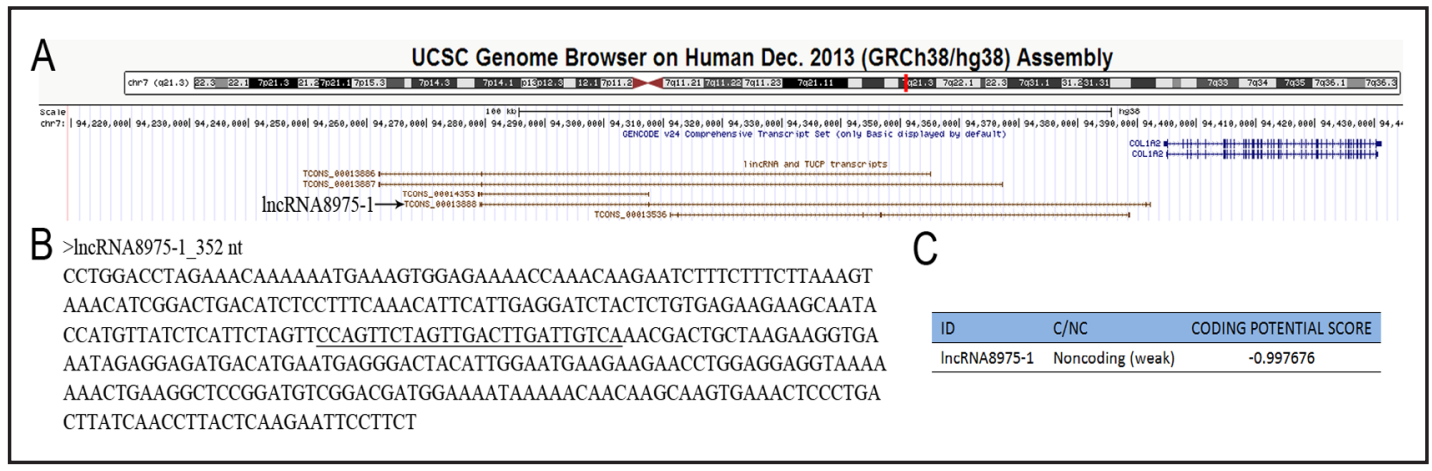

Fig. 2. Characteristics of IncRNA8975-1. (A) Chromosome location of IncRNA897-1 and information of the nearest gene based on the UCSC hg38 database. (B) Transcript of lncRNA8975-1 from the UCSC hg38 database. (C) The Coding Potential Calculator result indicates that lncRNA8975-1 is a non-coding RNA.

\section{KARGER}




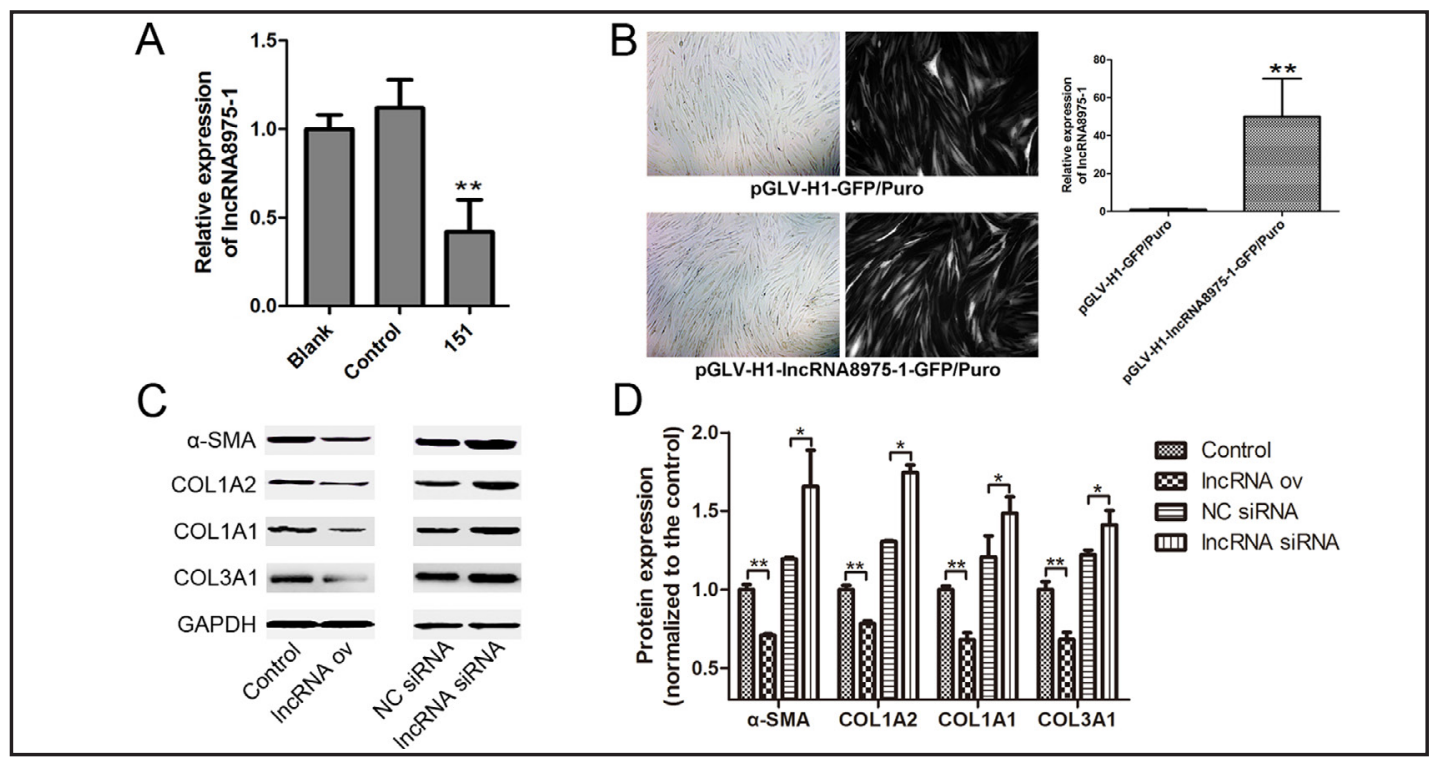

Fig. 3. Effects of knocking down or overexpressing lncRNA8975-1 on collagen synthesis and $\alpha$-SMA in HSFs. Expression of lncRNA8975-1 was detected by qRT-PCR after siRNA transfection (A) or lentiviral infection (B). (C) Western blot analysis was used to evaluate the protein expression of $\alpha$-SMA, COL1A2, COL1A1 and COL3A1. (D) Densitometry analysis of western blot results. ${ }^{*} P<0.05$, ${ }^{* *} P<0.01$, versus either the control or NC siRNA groups. Control: overexpression of an empty negative control vector (pGLV-H1-GFP/Puro); lncRNA ov: overexpression of the 352 bp IncRNA8975-1 transcript (pGLV-H1- IncRNA8975-1-GFP/Puro); NC siRNA: Stealth RNAi ${ }^{\mathrm{TM}}$ siRNA Negative Control; IncRNA siRNA: Stealth_151 duplexes against lncRNA8975-1.

and is a natural antisense lncRNA (Fig. 2A, 2B). Consistent with lncRNA8975-1 being a noncoding RNA in the UCSC database, the CPC (Coding Potential Calculator) computational algorithm (http://cpc.cbi.pku.edu.cn/programs/run_cpc.jsp) [15] predicted that lncRNA8975-1 has very low coding potential (Fig. 2C) similar to MEG9, a well-documented bovine lncRNA with coding potential score of -0.80266 [16].

\section{LncRNA8975-1 affects collagen and $\alpha$-SMA expression in fibroblasts}

We then selected HSF cells for knockdown and overexpression experiments. The expression of lncRNA8975-1 was knocked down after transfection with a specific siRNA (Stealth_151 siRNA, 151). Overexpression of lncRNA8975-1 was performed using lentiviral infection with the 352 nt lncRNA8975-1. Four lncRNA and TUCP transcripts are adjacent to IncRNA8975-1 and the COL1A2 gene (Fig. 2A). We therefore used nucleotide BLAST and Vector NTI sequence alignment to check that the Stealth_151 siRNA is specific for lncRNA8975-1. As shown in Fig. 3A, after $96 \mathrm{~h}$ of treatments in HSF cells, the expression of lncRNA8975-1 was significantly reduced to $42 \%$ of the control levels after transfecting HSF cells with stealth_151 siRNA, whereas lentiviral infection led to the overexpression of lncRNA8975-1 in HSF cells (Fig. 3B).

It is known that antisense RNAs usually regulate their counterpart sense mRNA [17]. The relationship between lncRNA8975-1 and COL1A2 is natural antisense (Fig. 2A). Therefore, we measured COL1A2 expression in fibroblasts with overexpression or knockdown of IncRNA8975-1. Moreover, hypertrophic scars are related to abundant alpha-smooth muscle actin $(\alpha-S M A)$-producing myofibroblasts in conjunction with increased levels of type III collagen and type I collagen [6]. Thus, we also determined whether the expression of COL1A1, COL3A1 and $\alpha$-SMA were changed in fibroblasts with overexpression or knockdown of IncRNA8975-1. Interestingly, western blot analysis showed that the protein expression of COL1A2, COL1A1, COL3A1 and $\alpha$-SMA were all reduced in the lncRNA8975-1 overexpressing group than in control HSF cells (Fig. 3C, 3D). However, knocking down lncRNA8975-1 increased the protein expression levels of COL1A2, COL1A1, COL3A1 and $\alpha$-SMA in HSF 


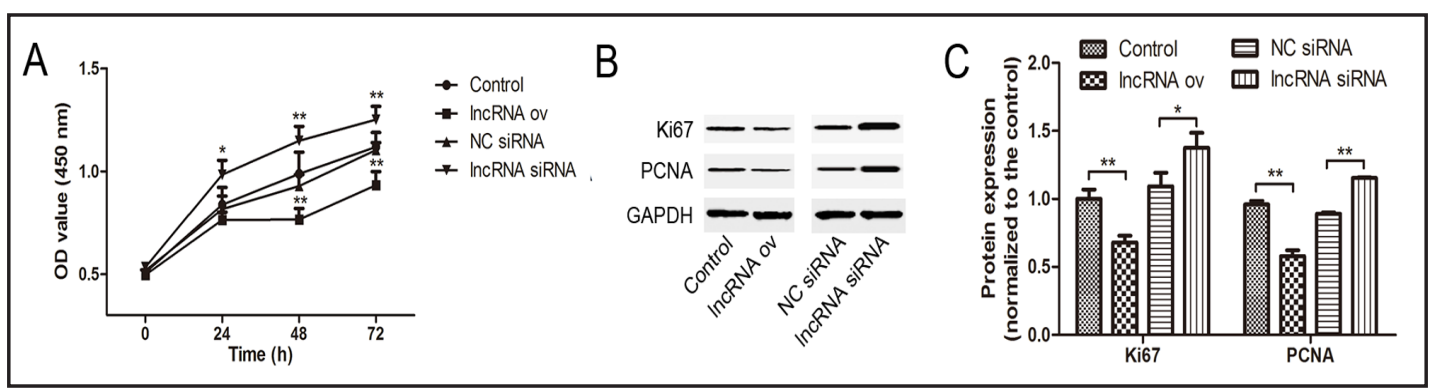

Fig. 4. Effects of knocking down or overexpressing lncRNA8975-1 on fibroblast proliferation and fibroblastrelated proliferation markers. (A) Cell proliferation was measured by using the CCK-8 assay. (B) Western blot analysis was used to evaluate the expression of Ki67 and PCNA in HSFs. (D) Densitometry analysis of western blot results. ${ }^{*} P<0.05$, ${ }^{* *} P<0.01$, versus either the control or NC siRNA groups. Control: overexpression of an empty negative control vector (pGLV-H1-GFP/Puro); IncRNA ov: overexpression of the 352 bp IncRNA8975-1 transcript (pGLV-H1- IncRNA8975-1-GFP/Puro); NC siRNA: Stealth RNAi ${ }^{\text {TM }}$ siRNA Negative Control; IncRNA siRNA: Stealth_151 duplexes against lncRNA8975-1.

cells (Fig. 3C, 3D). Together, these data suggest that dysregulation of lncRNA8975-1 affects collagen and $\alpha$-SMA expression in fibroblasts.

\section{LncRNA8975-1 alters cell proliferation in fibroblasts}

We further assessed the effects of IncRNA8975-1 on cell proliferation. CCK-8 was used to measure cell proliferation. It was observed that knockdown of lncRNA8975-1 significantly increased cell proliferation, whereas overexpression of lncRNA8975-1 inhibited HSF cell proliferation (Fig. 4A). Western blot analysis showed that the cell proliferation markers Ki67 and PCNA were all increased in HSF cells with lncRNA8975-1 knockdown but decreased in HSF cells over expressing lncRNA8975-1 (Fig. 4B, 4C). Taken together, our data revealed that dysregulation of lncRNA8975-1 alters cell proliferation in fibroblasts.

\section{Discussion}

LncRNAs play crucial roles in multiple developmental processes and diseases at almost every regulatory level of gene expression $[18,19]$. Hypertrophic scar formation is an abnormal sign of wound healing with sophisticated aetiology. The role of lncRNAs in hypertrophic scar formation has been poorly investigated. Our study found that lncRNA8975-1 was significantly overexpressed in hypertrophic scar tissues and fibroblasts. These results demonstrated that lncRNA8975-1 is involved in fibroblast proliferation and collagen synthesis and therefore contributes to the pathogenesis of hypertrophic scars.

A recent study reported that IncRNA8975-1 (TCONS_00013888) was disrupted in severe speech and language disorders [20]. Our group previously showed that lncRNA8975-1 was overexpressed in regressive scars compared to mature scar tissue [12]. In this study, by using lncRNA8975-1 lentivirus infection and siRNA transfection strategies, we found that lncRNA8975-1 inhibited fibroblast proliferation as well as collagen and $\alpha$-SMA expression. These findings suggest that elevated expression of lncRNA8975-1 in hypertrophic scars may inhibit collagen synthesis. However, overexpression of $\alpha$-SMA and collagen happens in hypertrophic scaring. One explanation is that overexpression of lncRNA8975-1 in HSF cells using lentivirus infection caused more lncRNA8975-1 than the upregulation of IncRNA8975-1 in hypertrophic scar tissues. Therefore, more overexpression of lncRNA8975-1 could inhibit collagen and $\alpha$-SMA expression. Another reason could be that higher expression of lncRNA8975-1 is a result but not the cause of hypertrophic scar formation. Therefore, even lncRNA8975-1 inhibits $\alpha$-SMA or collagen expression, other fibrotic factors such as connective tissue growth factors, Th2 cytokines and insulin-like growth factor-1 (IGF-1) could increase collagen synthesis and $\alpha$-SMA expression [1] bypass the role of IncRNA8975-1.

\section{KARGER}


Excess deposition of extracellular matrix proteins particularly type I collagen (which consists of a1 (I) and a2 (I) collagen) and type III collagen by fibroblasts is responsible for hypertrophic scar formation [21]. One previous study reported that the expression of type I and III collagen was found to be significantly higher in HS tissues and HSFs than in NS tissues and NSFs [22]. In humans, imbalanced cell proliferation of HSFs would cause hypertrophic scar formation [21]. Our study found for the first time that the long non-coding RNA lncRNA8975-1 inhibited cell proliferation as well as collagen and $\alpha$-SMA expression in hypertrophic scar fibroblasts.

Antisense lncRNAs usually regulate their counterpart sense mRNAs in cis by bridging epigenetic effectors and regulatory complexes at specific genomic loci [17]. It is known that the proximal promoter of COL1A2 is under the control of several transcription factors and epigenetic effectors [23]. A repressor complex that contains RFX5, HDAC2, Sin3B, and G9a is responsible for IFN- $\gamma$-induced COL1A2 repression in vascular smooth muscle cells [24]. In our study, the relationship between IncRNA8975-1 and COL1A2 is natural antisense. It is possible that lncRNA8975-1 may recruit the transcriptional repressor complex to the COL1A2 promoter to inhibit COL1A2 expression. Transforming growth factor (TGF)-beta signalling could promote collagen expression and induce the differentiation of fibroblasts into myofibroblasts $[1,25]$. Thus, lncRNA8975-1 may inhibit TGF-beta signalling and reduce COL1A1, COL3A1 and $\alpha$-SMA expression. However, the molecular mechanism involved requires further study.

MicroRNAs play crucial roles in skin fibrosis [26]. The proliferation of hypertrophic scar fibroblasts was reported to be affected by the non-coding RNAs miR-200b, miR-21 and miR-143-3p [27-29]. The functional interactions of IncRNAs, miRNAs and mRNAs could lead to a new explanation for the pathogenesis of keloids [8]. We used DIANA-LncBase v2 (www.microrna.gr/LncBase) [30], LNCipedia v4.0 (www.lncipedia.org) [31] and NPInter v3.0 (www.bioinfo.org/NPInter/) [32] to determine whether any miRNAs could associate with IncRNA8975-1. Unfortunately, we did not find any miRNAs; however, using a miRBase sequencing matching strategy (www.mirbase.org/search.shtml) as described in the literature [33], we found that IncRNA8975-1 contains potential binding sites for 9 miRNAs: miR-1273a, miR-30e-3p, miR-2681-5p, miR-30d-3p, miR-3925-3p, miR-4775, miR-67395p, miR-491-3p, and miR-3653-5p. Whether lncRNA8975-1 interacts with these microRNAs to regulate fibroblast activity also needs further exploration.

Interestingly, gene polymorphisms play crucial roles in the pathogenesis of scarring $[34,35]$. Further elucidating the underlying mechanism of the function of lncRNA8975-1 in hypertrophic scarring would be helpful in revealing the biological aetiology and potentially providing useful information for scar evaluation and treatments.

\section{Acknowledgements}

This study was supported by the National Natural Science Foundation of China (81501672); the Natural Science Foundation of Jiangsu Province (BK20140083); the Nanjing Medical Science and Technique Development Foundation (YKK15159); and the Nanjing Science and Technology project (201503047).

\section{Disclosure Statement}

The authors declare that they have no conflict of interests.

\section{References}

1 Zhu Z, Ding J, Shankowsky HA, Tredget EE: The molecular mechanism of hypertrophic scar. J Cell Commun Signal 2013;7:239-252. 


\section{Cellular Physiology Cell Physiol Biochem 2016;40:326-334

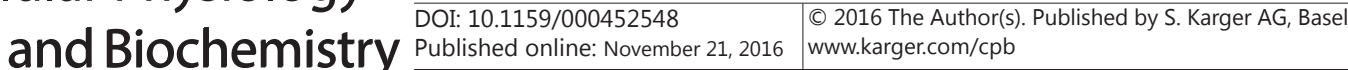 \\ Li et al.: LncRNA Controls Collagen Expression}

2 Bombaro KM, Engrav LH, Carrougher GJ, Wiechman SA, Faucher L, Costa BA, Heimbach DM, Rivara FP, Honari S: What is the prevalence of hypertrophic scarring following burns? Burns 2003;29:299-302.

3 Rabello FB, Souza CD, Farina Junior JA: Update on hypertrophic scar treatment. Clinics (Sao Paulo) 2014;69:565-573.

4 Zhang Z, Li XJ, Liu Y, Zhang X, Li YY, Xu WS: Recombinant human decorin inhibits cell proliferation and downregulates TGF-beta1 production in hypertrophic scar fibroblasts. Burns 2007;33:634-641.

5 Tredget EE, Levi B, Donelan MB: Biology and principles of scar management and burn reconstruction. Surg Clin North Am 2014;94:793-815.

6 Arno AI, Gauglitz GG, Barret JP, Jeschke MG: Up-to-date approach to manage keloids and hypertrophic scars: a useful guide. Burns 2014;40:1255-1266.

7 Yarmishyn AA, Kurochkin IV: Long noncoding RNAs: a potential novel class of cancer biomarkers. Front Genet 2015;6:145.

8 Zhu HY, Bai WD, Li C, Zheng Z, Guan H, Liu JQ, Yang XK, Han SC, Gao JX, Wang HT, Hu DH: Knockdown of lncRNA-ATB suppresses autocrine secretion of TGF-beta2 by targeting ZNF217 via miR-200c in keloid fibroblasts. Sci Rep 2016;6:24728.

9 Wang Z, Jinnin M, Nakamura K, Harada M, Kudo H, Nakayama W, Inoue K, Nakashima T, Honda N, Fukushima S, Ihn H: Long non-coding RNA TSIX is upregulated in scleroderma dermal fibroblasts and controls collagen mRNA stabilization. Exp Dermatol 2016;25:131-136.

10 Sun H, Chen J, Qian W, Kang J, Wang J, Jiang L, Qiao L, Chen W, Zhang J: Integrated long non-coding RNA analyses identify novel regulators of epithelial-mesenchymal transition in the mouse model of pulmonary fibrosis. J Cell Mol Med 2016;20:1234-1246.

11 Zhou C, York SR, Chen JY, Pondick JV, Motola DL, Chung RT, Mullen AC: Long noncoding RNAs expressed in human hepatic stellate cells form networks with extracellular matrix proteins. Genome Med 2016;8:31.

12 Li J, Long W, Li Q Zhou Q, Wang Y, Wang H, Zhou B, Li J: Distinct expression profiles of IncRNAs between regressive and mature scars. Cell Physiol Biochem 2015;35:663-675.

13 Arakawa M, Hatamochi A, Takeda K, Ueki H: Increased collagen synthesis accompanying elevated m-RNA levels in cultured Werner's syndrome fibroblasts. J Invest Dermatol 1990;94:187-190.

14 Larionov A, Krause A, Miller W: A standard curve based method for relative real time PCR data processing. BMC Bioinformatics 2005;6:62.

15 Kong L, Zhang Y, Ye ZQ Liu XQ, Zhao SQ, Wei L, Gao G: CPC: assess the protein-coding potential of transcripts using sequence features and support vector machine. Nucleic Acids Res 2007;35:W345-349.

16 Li M, Sun X, Cai H, Sun Y, Plath M, Li C, Lan X, Lei C, Lin F, Bai Y, Chen H: Long non-coding RNA ADNCR suppresses adipogenic differentiation by targeting miR-204. Biochim Biophys Acta 2016;1859:871-882.

17 Magistri M, Faghihi MA, St Laurent G, 3rd, Wahlestedt C: Regulation of chromatin structure by long noncoding RNAs: focus on natural antisense transcripts. Trends Genet 2012;28:389-396.

18 Takahashi K, Yan I, Haga H, Patel T: Long noncoding RNA in liver diseases. Hepatology 2014;60:744-753.

19 Fatica A, Bozzoni I: Long non-coding RNAs: new players in cell differentiation and development. Nat Rev Genet 2014;15:7-21.

20 Moralli D, Nudel R, Chan MT, Green CM, Volpi EV, Benitez-Burraco A, Newbury DF, Garcia-Bellido P: Language impairment in a case of a complex chromosomal rearrangement with a breakpoint downstream of FOXP2. Mol Cytogenet 2015;8:36.

21 Fang F, Huang RL, Zheng Y, Liu M, Huo R: Bone marrow derived mesenchymal stem cells inhibit the proliferative and profibrotic phenotype of hypertrophic scar fibroblasts and keloid fibroblasts through paracrine signaling. J Dermatol Sci 2016;83:95-105.

22 Zhao J, Shu B, Chen L, Tang J, Zhang L, Xie J, Liu X, Xu Y, Qi S: Prostaglandin E inhibits collagen synthesis in dermal fibroblasts and prevents hypertrophic scar formation in vivo. Exp Dermatol 2016;25:604-610.

23 Ramirez F, Tanaka S, Bou-Gharios G: Transcriptional regulation of the human alpha2(I) collagen gene (COL1A2), an informative model system to study fibrotic diseases. Matrix Biol 2006;25:365-372.

24 Weng X, Cheng X, Wu X, Xu H, Fang M, Xu Y: Sin3B mediates collagen type I gene repression by interferon gamma in vascular smooth muscle cells. Biochem Biophys Res Commun 2014;447:263-270.

25 He T, Bai X, Yang L, Fan L, Li Y, Su L, Gao J, Han S, Hu D: Loureirin B Inhibits Hypertrophic Scar Formation via Inhibition of the TGF- $\beta 1$-ERK/JNK Pathway. Cell Physiol Biochem 2015;37:666-676.

26 Babalola 0, Mamalis A, Lev-Tov H, Jagdeo J: The role of microRNAs in skin fibrosis. Arch Dermatol Res 2013;305:763-776. 


\section{Cellular Physiology Cell Physiol Biochem 2016;40:326-334 \begin{tabular}{ll|l} 
DOI: 10.1159/000452548 & $\begin{array}{l}\text { O 2016 The Author(s). Published by S. Karger AG, Basel } \\
\text { www.karger.com/cpb }\end{array}$
\end{tabular} \\ Li et al.: LncRNA Controls Collagen Expression}

27 Li P, He QY, Luo CQ: Overexpression of miR-200b inhibits the cell proliferation and promotes apoptosis of human hypertrophic scar fibroblasts in vitro. J Dermatol 2014;41:903-911.

28 Mu S, Kang B, Zeng W, Sun Y, Yang F: MicroRNA-143-3p inhibits hyperplastic scar formation by targeting connective tissue growth factor CTGF/CCN2 via the Akt/mTOR pathway. Mol Cell Biochem 2016;416:99108.

29 Zhu HY, Li C, Bai WD, Su LL, Liu JQ Li Y, Shi JH, Cai WX, Bai XZ, Jia YH, Zhao B, Wu X, Li J, Hu DH: MicroRNA-21 regulates hTERT via PTEN in hypertrophic scar fibroblasts. PLoS One 2014;9:e97114.

30 Paraskevopoulou MD, Vlachos IS, Karagkouni D, Georgakilas G, Kanellos I, Vergoulis T, Zagganas K, Tsanakas P, Floros E, Dalamagas T, Hatzigeorgiou AG: DIANA-LncBase v2: indexing microRNA targets on non-coding transcripts. Nucleic Acids Res 2016;44:D231-238.

31 Volders PJ, Helsens K, Wang X, Menten B, Martens L, Gevaert K, Vandesompele J, Mestdagh P: LNCipedia: a database for annotated human lncRNA transcript sequences and structures. Nucleic Acids Res 2013;41:D246-251.

32 Hao Y, Wu W, Li H, Yuan J, Luo J, Zhao Y, Chen R: NPInter v3.0: an upgraded database of noncoding RNAassociated interactions. Database (Oxford) DOI:10.1093/database/baw057.

33 Gao Y, Meng H, Liu S, Hu J, Zhang Y, Jiao T, Liu Y, Ou J, Wang D, Yao L, Liu S, Hui N: LncRNA-HOST2 regulates cell biological behaviors in epithelial ovarian cancer through a mechanism involving microRNA let-7b. Hum Mol Genet 2015;24:841-852.

34 Han J, Han J, Yu D, Xiao J, Shang Y, Hao L: Association of ADAM33 gene polymorphisms with keloid scars in a northeastern Chinese population. Cell Physiol Biochem 2014;34:981-987.

35 Yu D, Shang Y, Luo S, Hao L: The TaqI gene polymorphisms of VDR and the circulating 1,25-dihydroxyvitamin D levels confer the risk for the keloid scarring in Chinese cohorts. Cell Physiol Biochem 2013;32:39-45. 